\title{
Simplicity, in Mathematics and in Art
}

\author{
Allyn Jackson
}

Simplicity is as hard to pinpoint in mathematics as it is in art. Certainly both subjects have their great exemplars of the quality. But is there a definition of simplicity? A criterion? A measure? Or a sure path to it?

These kinds of questions were in the air at a conference called Simplicity: Ideals of Practice in Mathematics and the Arts, which took place at the Graduate Center of the City University of New York in early April 2013. Instead of trying to definitively answer such questions-surely a doomed prospect anyway-the participants gave in to the sheer joy of discussion in the stimulating atmosphere of each other's company. The conference featured lectures and panel discussions by an eclectic group of twenty-five artists, architects, art historians, mathematicians, and mathematically inclined philosophers, as well as a film program. The audience included academics from nearby institutions and local artists; as the conference offered easy and free online registration, a random smattering of folks wandered in out of curiosity.

\section{Not an Absolute Notion}

Simplicity often seems to be a timeless, absolute quality, and for good reason. Peter Sarnak, Institute for Advanced Study and Princeton University, offered Euclid's proof of the infinitude of primes as simplicity par excellence. The stark elegance of this ancient proof is as striking today as it must have been to people encountering it through the millennia. Of course, the proof is an exemplar of simplicity, not a definition. Indeed, Curtis Franks, University of Notre Dame, argued against the

Allyn Jackson is senior writer and deputy editor of the Notices. Her email address is axj@ams . org.

DOI: http://dx.doi.org/10.1090/noti1028 possibility of ever establishing for all time an absolute notion of simplicity. What we think of as simple emerges from conventions that are deeply embedded in how we live and how we see the world, and they have a long genetic history. "Our thinking occurs within those conventions," he said. "There is not really a way out of them."

As conventions evolve, so do notions of simplicity. Franks mentioned Gauss's 1831 paper that established the respectability of complex numbers. The problem Gauss was working on-concerning quadratic and biquadratic residues-had only unsatisfyingly complicated and piecemeal solutions over $\mathbb{Z}$. Over $\mathbb{C}$, a far simpler solution emerged. The complex numbers revealed simplicity where previously there had seemed to be none.

Mathematics is not engaged in a straightforward march toward absolute simplicity. Rather, by discovering simplicity anew, Franks said, "We will be more awake to the changing landscape of mathematical thought." He noted a parallel in art, where something new-like the work of Andy Warhol or Marcel Duchamp-acts as a sort of "shock treatment" that compels a new perspective.

Several conference speakers mentioned the art of Fred Sandback, who used taut lengths of yarn to represent outlines of three-dimensional shapes. In photos, the works look unimpressive; as philosopher Juliet Floyd, Boston University, noted, they are "unphotographable". But walking around and through the constructions, she found them to be "extremely moving objects". Finnish architect Juhani Pallasmaa described how a Sandback construction, merely "a few lines stretched in space", sets off a chain reaction in the viewer's mind, causing the viewer to see figures of specific material shapes, to feel their weight and texture. "The air inside the imaginary figure seems to get 
denser and to have a slightly different consistency from the air outside," he said. Simple constructions that hold much complexity and meaning: That's just what mathematicians seek in their work.

Pallasmaa's erudite lecture contained many striking quotations, including this one of Balthus: "The more anonymous painting is, the more real it is." The same can be said for architecture, Pallasmaa stated. Could a similar statement be made for mathematics? Are there mathematical results that are so natural, so pristine that one cannot perceive the fingerprints of the mathematicians who discovered them? Perhaps one example would be the previously mentioned proof of the infinitude of primes, its attribution to Euclid notwithstanding. Perhaps others are found in what Paul Erdős famously called "proofs from the Book".

Pallasmaa also quoted the philosopher Gaston Bachelard, who in his book The Philosophy of No: A Philosophy of the New Scientific Mind, stated that scientific thought "develops along a predestined path, from animism through realism, rationalism, and complex rationalism, to dialectical rationalism." Pallasmaa did not say that mathematics develops in this way; his point rather was that art aspires to develop in the opposite direction, from the rational back towards "a unifying, mythical, and animistic experience”. Perhaps mathematics shuttles back and forth between the two endpoints.

\section{Visceral Encounters}

Bachelard's "predestined path" at times echoed through the conference in comments that seemed to derive from the misconception, common outside of mathematics, that the subject consists entirely of proofs, progressing inexorably from one logical step to the next. This misconception was vividly countered at various points during the conference. In an open microphone session, Blaise Heltai pointed out that mathematics and art are actually very similar in process: When you are thinking about a mathematical object, you are right inside the thing, trying to puzzle out its structure and secrets. You're not thinking about how to prove anything-that comes later. The puzzling-out resembles the conceptual part of doing art. Heltai has a special perspective, as he is a painter with a Ph.D. in mathematics; he makes a living as a management consultant.

The kind of visceral encounter with mathematics that Heltai referred to emerged at various times, such as in the lecture of Dennis Sullivan, CUNY Graduate Center and Stony Brook University. When as a graduate student he was preparing for the preliminary examination, Sullivan studied John Milnor's book Topology from the Differential Viewpoint. Sullivan knew the book inside and out, every definition, every proof. The day before the exam, as he took a final glance through the book, it suddenly occurred to him that he could compress the contents into a single, simple picture. Moving back and forth across the stage, he used gesticulations to indicate a 2-sphere on one side, a 3 -sphere on the other, and a "slinky" curve between them. This curve, representing the preimage of a regular value of a map from the 3-sphere to the 2sphere, provided a mental image summarizing the Pontryagin-Thom construction. If one knows the language of manifolds and transversality, Sullivan claimed, one can reconstruct the whole theory of cobordism in differential topology just from the intuition conveyed by his slinky picture. This experience made him realize, "That's what it means to understand a piece of mathematics."

The visceral component of mathematical work surely evokes strong feelings, but mathematicians usually do not discuss their feelings about their work, at least not in public lectures. In an earlier panel discussion, Riikka Stewen, Finnish Academy of Fine Arts, asked whether mathematicians have strong love/hate feelings about their work. "Yes, very strong feelings," came the immediate reply from a mathematician on the panel, Andrés Villaveces, National University of Colombia. There is a loneliness in the work of an artist, and much mathematical work shares this quality. Just as a painter faces an empty canvas, he said, "Mathematicians are up against the empty page every day."

The longing, even desperation, that is implicit in the remarks of Villaveces also emerged in Sarnak's lecture, titled "Is there a place for 'ugly' mathematics?". Sarnak considered the situation where the only known route to a proof is ugly, in the sense of being strewn with long and complicated calculations and verifications. The question then becomes, How desperate are we for a proof? When Sarnak gave an example of an ugly calculation connected with a beautiful result in the theory of automorphic forms, Mikhail Gromov, Institut des Hautes Études Scientifiques and New York University, piped up to say: "Maybe the mathematics is fine, it's your mind that's ugly."

Then there was Gromov's lecture. A fish says: "You want to understand what water is? Jump in and find out." Instead of plunging in, you could study the chemical and physical properties of water. But without the experience of plunging into water, you have no frame in which to talk about what water really is. Similarly, when the experience of plunging into mathematics is absent, there is no frame in which to talk about what mathematics is-much less what simplicity in mathematics is.

That's a verbose description of one moment that flashed by in an instant in Gromov's stream-ofconsciousness lecture. He jumped into Descartes's timeless statement, "Cogito ergo sum [I think 
therefore I am]". The important thing here, Gromov said, is the ergo, the therefore. In a sense, dogs think: Much of what goes on in a human brain is very similar to what goes on in the brain of a dog. Surely dogs are. But dogs do not understand ergo. This ergo is a major source of the kind of thinking that is characteristic of humans, Gromov said. And yet, "it is completely hidden from us. And there is a good reason why it is hidden. If it surfaces, you die. You will not survive. It's against survival, it's against evolution, it's against [natural] selection."

So it went. Gromov passed so quickly over so many topics, diving to the depths, all the while leavening the presentation with flashes of subversive humor. The effect was dizzying. Afterward, during the open microphone session, an audience member demanded a one-sentence summary - with an example. An impossible request to fulfill. Nevertheless it can be said that one of Gromov's main messages was: Guard against the delusion of false simplicity. Many things that we assume at first glance to be simple are in fact highly complex.

After seeing Gromov's effervescent mind bubble over for thirty minutes, audience member Al Thaler, known to many for his long service at the National Science Foundation and now an adjunct faculty member at CUNY's Hunter College, commented, "I could never live like that."

\section{Contrasting Groups}

The Simplicity conference was the brainchild of mathematician Juliette Kennedy, University of Helsinki, and two CUNY mathematicians, Roman Kossak of the Graduate Center and Philip Ording of Medgar Evers College. The conference was something of a follow-up to a 2007 symposium called Aesthetics and Mathematics, which took place in Utrecht and was organized by Kennedy and two University of Utrecht mathematicians, Rosalie Iemhoff and Albert Visser (Iemhoff was one of the lecturers at Simplicity). Participants in the 2007 symposium could drop in at an art exhibition at the Mondriaanhuis, Logic Unfettered-European and American Abstraction Now, which was curated by Kennedy.

In addition to the film program at the Simplicity conference, there was an installation of a few works by artist Kate Shepherd in the lobby outside the hall where the lectures were given (Shepherd also participated in one of the panel discussions). But space constraints there, as well as the difficulty of securing exhibit space in New York City, meant that Simplicity offered few opportunities to experience art. As a result, art was represented mainly through the presence and words of the artists themselves. By contrast, the mathematicians could actually present pieces of mathematics by using a computer and a beamer, or even just a blackboard, in the case of Sullivan. They tried mightily to avoid technical details, with imperfect success.

Another contrast was socio-economic. As Kennedy pointed out in a panel discussion, the mathematicians and philosophers at the conference all work in academia, which provides economic security and social acceptability, while artists often lead far more precarious lives on the fringes of society. She noted the "heroic" efforts that many artists must put forth in order to carry out their work.

What did each group absorb from the other? It's difficult to say. One participant observed that mathematicians tend to have a high opinion of themselves and their own knowledge and are therefore not so open to new ideas, while artists are pretty much the opposite: Receptiveness to impressions and influences from a wide variety of sources is the artist's lifeblood. One artist who attended Simplicity, Miyuki Tsushima, said she didn't follow all the details of the math lectures. She could simply sit and let the impressions wash over her as she made some sketches for her latest work.

An inspiration for the conference was the socalled twenty-fourth problem of David Hilbert. This problem, which Hilbert considered adding to his famous list of twenty-three problems that he presented at the International Congress of Mathematicians in Paris in 1900, was unearthed by Rüdiger Thiele, University of Leipzig, from papers at the library of the University of Göttingen. Part of Hilbert's description of the problem reads: "Criteria of simplicity, or proof of the greatest simplicity of certain proofs. Develop a theory of the method of proof in mathematics in general. Under a given set of conditions there can be but one simplest proof" (translation by Thiele from his article "Hilbert's 24th Problem", American Mathematical Monthly, January 2003).

Etienne Ghys, École Normale Supérieure de Lyon, pointed out the naiveté of imagining that such ultimate simplicity is possible. Yet, as the conference highlighted, simplicity as a dream, as an ideal, remains a powerful guiding light in mathematics and the arts. As Franks said, there are no absolute notions of simplicity. But do not relinquish the quest. "On the contrary, I want to say yes, find criteria for simplicity, continue to do so," said Franks. Don't imagine that the matter will ever be settled definitively; rather, "return to the task often."

Materials from some of the lectures are on the Simplicity conference website, http://s-i-m-p-1$\mathrm{i}-\mathrm{c}-\mathrm{i}-\mathrm{t}-\mathrm{y} . \mathrm{org}$, and videos of some of the lectures will be posted soon. 